TITLE:

\title{
Critical earthquake input energy to connected building structures using impulse input
}

$\operatorname{AUTHOR}(\mathrm{S})$ :

Fukumoto, Yoshiyuki; Takewaki, Izuru

CITATION:

Fukumoto, Yoshiyuki ... [et al]. Critical earthquake input energy to connected building structures using impulse input. Earthquake and Structures 2015, 9(6): 1133-1152

ISSUE DATE:

2015-12-25

URL:

http://hdl.handle.net/2433/241753

RIGHT:

許諾条件に基づいて掲載しています。; This is not the published version. Please cite only the published version. この論文は出版社版で ありません。引用の際には出版社版をご確認ご利用ください 


\title{
Critical earthquake input energy to connected building structures using impulse input
}

\author{
Yoshiyuki Fukumoto and Izuru Takewaki* \\ Department of Architecture and Architectural Engineering, Kyoto University \\ Kyotodaigaku-Katsura, Nishikyo, Kyoto 615-8540, Japan \\ *Corresponding author, E-mail: takewaki@ archi.kyoto-u.ac.jp
}

\begin{abstract}
A frequency-domain method is developed for evaluating the earthquake input energy to two building structures connected by viscous dampers. It is shown that the earthquake input energies to respective building structures and viscous connecting dampers can be defined as works done by the boundary forces between the subsystems on their corresponding displacements. It is demonstrated that the proposed energy transfer function is very useful for clear understanding of dependence of energy consumption ratios in respective buildings and connecting viscous dampers on their properties. It can be shown that the area of the energy transfer function for the total system is constant regardless of natural period and damping ratio because the constant Fourier amplitude of the input acceleration, relating directly the area of the energy transfer function to the input energy, indicates the Dirac delta function and only an initial velocity (kinetic energy) is given in this case. Owing to the constant area property of the energy transfer functions, the total input energy to the overall system including both buildings and connecting viscous dampers is approximately constant regardless of the quantity of connecting viscous dampers. This property leads to an advantageous feature that, if the energy consumption in the connecting viscous dampers increases, the input energies to the buildings can be reduced drastically. For the worst case analysis, critical excitation problems with respect to the impulse interval for double impulse (simplification of pulse-type impulsive ground motion) and multiple impulses (simplification of longduration ground motion) are considered and their solutions are provided.
\end{abstract}

Keywords: earthquake input energy, frequency-domain analysis, time-domain analysis, energy transfer function, connected buildings, passive structural control, impulsive ground motion, long-duration ground motion, critical excitation 


\section{Introduction}

In the current structural design practice of buildings in earthquake prone countries, the structural control using base-isolation systems and passive dampers is of primary concern. Especially a hybrid system of a base-isolation system and a passive damper control system is getting much interest (for example Murase et al. 2013).

Since base-isolation systems and passive control dampers are generally characterized as systems to absorb considerable energies supplied by a ground motion input, it is believed that the investigation from the viewpoint of earthquake input energy is suitable in such systems. A lot of research works have been conducted on the topics of earthquake input energy to building structures (for example, Housner 1959; Zahrah and Hall 1984; Akiyama 1985; Uang and Bertero 1990; Leger and Dussault 1992; Kuwamura et al. 1994; Ordaz et al. 2003; Takewaki 2004a, b, 2005a, b, 2007a, b). In some earthquake prone countries, the earthquake input energy is treated as an earthquake input demand in the seismic design regulations. The earthquake input energy has usually been computed in the time domain since the development of advanced computational algorithms, e.g. numerical integration algorithms. The time-domain approach has several advantages, e.g. the availability in non-linear structures, the description of time-history response of the input energy, the possibility of expressing the input energy rate. On the other hand, the time-domain approach is not necessarily appropriate for probabilistic and bound analysis under uncertainties (Takewaki 2004a, 2005b, 2007). For such purpose, the frequency-domain approach (Lyon 1975, Ordaz et al. 2003, Takewaki 2004a, b, 2005a, b, 2006, 2007a, b) is appropriate because it uses the Fourier amplitude spectrum of input ground accelerations and the time-invariant energy transfer functions of the structure in an independent product form.

Vibration control of buildings by connection is very popular recently (Seto 1994, Luco and Barros 1998, Xu et al. 1999, Kim et al. 2006, Takewaki 2007b, Christenson et al. 2007, Basili and Angelis 2007, Ok et al. 2008, Cimellaro et al. 2011, Roh et al. 2011, Zhu et al. 2011, Richardson et al. 2013a, b). It was pointed out that the use of the difference of natural periods of two buildings is very useful and effective for vibration suppression because no energy supply is necessary. It was shown that the total input energy by actual ground motions to the overall system including both buildings and connecting viscous dampers is supposed to be almost constant regardless of the quantity and location of connecting viscous dampers. This property was partially examined by the use of two single-degree-of-freedom (SDOF) models connected by a viscous damper. A remarkable property of equi-area of the energy transfer functions supports the property of the nearly constant input energy. This leads to an advantageous feature that, if the energy consumption in the connecting viscous dampers increases, the input energies to the buildings can be reduced drastically.

In this paper, the input energies to connected buildings under double impulse (simplification of pulse-type impulsive ground motion) and multiple impulses (simplification of long-period and long-duration ground motion) are considered. Critical excitation problems for both inputs with respect to the impulse interval are posed and solved. 


\section{Earthquake input energy to connected SDOF models}

\subsection{Equations of motion}

Consider a connected building structure shown in Fig.1(a). The right building is called building 1 (main structure) and the left one building 2 (sub-structure). It is noted that, when the spring and viscous damper in building 2 are removed, the model is reduced to a $2 \mathrm{DOF}$ shear building model. Two cases, i.e. a structural system of disconnected two SDOF models and a structural system of rigidly connected two SDOF models (Figs.1(b), (c)), have been treated in Reference (Takewaki 2007b) as limiting cases for clear understanding of vibration properties of this system. The formulation in the frequency domain will be shown in this section.

Let $m_{1}$ and $m_{2}$ denote the masses of building 1 and 2. The story stiffnesses and damping coefficients of building 1 and 2 are denoted by $k_{1}, c_{1}, k_{2}, c_{2}$, respectively. The stiffness and damping coefficient of the connecting damper are indicated by $k_{3}$ and $c_{3}$, respectively. The equations of motion of the model shown in Fig.1(a) may be expressed as

$$
\left[\begin{array}{cc}
m_{1} & 0 \\
0 & m_{2}
\end{array}\right]\left\{\begin{array}{l}
\ddot{u}_{1} \\
\ddot{u}_{2}
\end{array}\right\}+\left[\begin{array}{cc}
c_{1}+c_{3} & -c_{3} \\
-c_{3} & c_{2}+c_{3}
\end{array}\right]\left\{\begin{array}{l}
\dot{u}_{1} \\
\dot{u}_{2}
\end{array}\right\}+\left[\begin{array}{cc}
k_{1}+k_{3} & -k_{3} \\
-k_{3} & k_{2}+k_{3}
\end{array}\right]\left\{\begin{array}{l}
u_{1} \\
u_{2}
\end{array}\right\}=-\left[\begin{array}{cc}
m_{1} & 0 \\
0 & m_{2}
\end{array}\right]\left\{\begin{array}{l}
1 \\
1
\end{array}\right\} \ddot{u}_{g}
$$

Fourier transformation of Eq.(1) results in the following form.

$$
[G]\{U\}=\{B\} \ddot{U}_{g}(\omega)
$$

where $[G]$ and $\{B\}$ are the following coefficient matrix and vector.

$$
[G]=\left[\begin{array}{cc}
k_{1}+k_{3}-\omega^{2} m_{1}+\mathrm{i} \omega\left(c_{1}+c_{3}\right) & -k_{3}-\mathrm{i} \omega c_{3} \\
-k_{3}-\mathrm{i} \omega c_{3} & k_{2}+k_{3}-\omega^{2} m_{2}+\mathrm{i} \omega\left(c_{2}+c_{3}\right)
\end{array}\right]
$$

The vector $\{U\}=\left\{\begin{array}{ll}U_{1} & U_{2}\end{array}\right\}^{T}$ is the Fourier transform of the nodal displacements $\{u\}=\left\{\begin{array}{ll}u_{1} & u_{2}\end{array}\right\}^{T}$. i indicates the imaginary unit.

The transfer function of the nodal displacement with respect to $\ddot{U}_{g}(\omega)$ may be obtained as

$$
\left\{H_{D}\right\}=\left\{H_{D 1} \quad H_{D 2}\right\}^{T}=\left\{U_{1} / \ddot{U}_{g} \quad U_{2} / \ddot{U}_{g}\right\}^{T}=[G]^{-1}\{B\}
$$

where

$$
[G]^{-1}=\frac{1}{D}\left[\begin{array}{cc}
k_{2}+k_{3}-\omega^{2} m_{2}+\mathrm{i} \omega\left(c_{2}+c_{3}\right) & k_{3}+\mathrm{i} \omega c_{3} \\
k_{3}+\mathrm{i} \omega c_{3} & k_{1}+k_{3}-\omega^{2} m_{1}+\mathrm{i} \omega\left(c_{1}+c_{3}\right)
\end{array}\right]
$$


In Eq.(5), $D$ denotes the following

$$
\begin{aligned}
D= & \left\{k_{1}+k_{3}-\omega^{2} m_{1}+\mathrm{i} \omega\left(c_{1}+c_{3}\right)\right\}\left\{k_{2}+k_{3}-\omega^{2} m_{2}+\mathrm{i} \omega\left(c_{2}+c_{3}\right)\right\} \\
& -\left(k_{3}+\mathrm{i} \omega c_{3}\right)^{2} \\
= & a+b \mathrm{i}
\end{aligned}
$$

where

$$
\begin{aligned}
a= & m_{1} m_{2} \omega^{4}-\left(k_{1} m_{2}+k_{2} m_{1}+k_{3} m_{2}+k_{3} m_{1}+c_{1} c_{2}+c_{2} c_{3}+c_{3} c_{1}\right) \omega^{2} \\
& +k_{1} k_{2}+k_{2} k_{3}+k_{3} k_{1} \\
b= & -\left\{m_{1}\left(c_{2}+c_{3}\right)+m_{2}\left(c_{1}+c_{3}\right)\right\} \omega^{3} \\
& +\left\{\left(k_{1}+k_{3}\right)\left(c_{2}+c_{3}\right)+\left(k_{2}+k_{3}\right)\left(c_{1}+c_{3}\right)-2 k_{3} c_{3}\right\} \omega
\end{aligned}
$$

The transfer function can be obtained in closed form.

$$
\left\{H_{D}\right\}=-(1 / D)\{c+d \mathrm{i} \quad e+f \mathrm{i}\}^{T}
$$

where

$$
\begin{aligned}
& c=m_{1}\left(k_{2}+k_{3}-\omega^{2} m_{2}\right)+k_{3} m_{2} \\
& d=\left\{m_{1}\left(c_{2}+c_{3}\right)+m_{2} c_{3}\right\} \omega \\
& e=m_{2}\left(k_{1}+k_{3}-\omega^{2} m_{1}\right)+k_{3} m_{1} \\
& f=\left\{m_{2}\left(c_{1}+c_{3}\right)+m_{1} c_{3}\right\} \omega
\end{aligned}
$$

Finally the displacement and velocity transfer functions may be obtained in closed form.

$$
\begin{gathered}
\left\{\begin{array}{l}
H_{D 1} \\
H_{D 2}
\end{array}\right\}=-\frac{1}{a^{2}+b^{2}}\left\{\begin{array}{l}
a c+b d+(a d-b c) \mathrm{i} \\
a e+b f+(a f-b e) \mathrm{i}
\end{array}\right\} \\
\left\{\begin{array}{l}
H_{V 1} \\
H_{V 2}
\end{array}\right\}=\mathrm{i} \omega\left\{\begin{array}{l}
H_{D 1} \\
H_{D 2}
\end{array}\right\}=-\frac{1}{a^{2}+b^{2}}\left\{\begin{array}{l}
\mathrm{i} \omega(a c+b d)-\omega(a d-b c) \\
\mathrm{i} \omega(a e+b f)-\omega(a f-b e)
\end{array}\right\}
\end{gathered}
$$

\subsection{Earthquake input energy in frequency domain}

With the help of these closed-form expressions, the input energy to the overall model may be expressed as 


$$
\begin{aligned}
& E_{I} /\left(m_{1}+m_{2}\right)=\frac{1}{m_{1}+m_{2}}\left(-\int_{-\infty}^{\infty} m_{1} \dot{u}_{1} \ddot{u}_{g} d t-\int_{-\infty}^{\infty} m_{2} \dot{u}_{2} \ddot{u}_{g} d t\right) \\
& =-\frac{1}{m_{1}+m_{2}} \int_{-\infty}^{\infty}\left[(1 / 2 \pi) m_{1} \int_{-\infty}^{\infty} \dot{U}_{1} e^{\mathrm{i} \omega t} \mathrm{~d} \omega\right] \ddot{u}_{g} \mathrm{~d} t \\
& -\frac{1}{m_{1}+m_{2}} \int_{-\infty}^{\infty}\left[(1 / 2 \pi) m_{2} \int_{-\infty}^{\infty} \dot{U}_{2} e^{\mathrm{i} \omega t} \mathrm{~d} \omega\right] \ddot{u}_{g} \mathrm{~d} t \\
& =-\frac{1}{2 \pi\left(m_{1}+m_{2}\right)} \int_{-\infty}^{\infty}\left\{m_{1} \operatorname{Re}\left[H_{V 1}\right]+m_{2} \operatorname{Re}\left[H_{V 2}\right]\right\}\left|\ddot{U}_{g}(\omega)\right|^{2} d \omega \\
& =\frac{1}{2 \pi\left(m_{1}+m_{2}\right)} \int_{-\infty}^{\infty}\left\{\frac{m_{1} \omega(b c-a d)}{a^{2}+b^{2}}+\frac{m_{2} \omega(b e-a f)}{a^{2}+b^{2}}\right\}\left|\ddot{U}_{g}(\omega)\right|^{2} d \omega \\
& =\frac{1}{\pi\left(m_{1}+m_{2}\right)} \int_{0}^{\infty}\left\{\frac{m_{1} \omega(b c-a d)}{a^{2}+b^{2}}+\frac{m_{2} \omega(b e-a f)}{a^{2}+b^{2}}\right\}\left|\ddot{U}_{g}(\omega)\right|^{2} d \omega \\
& =\int_{0}^{\infty} F_{C}(\omega)\left|\ddot{U}_{g}(\omega)\right|^{2} d \omega
\end{aligned}
$$

where $F_{C}(\omega)$ is 'the energy transfer function' for the total system and is defined by

$$
F_{C}(\omega)=\frac{1}{\pi\left(m_{1}+m_{2}\right)}\left\{\frac{m_{1} \omega(b c-a d)}{a^{2}+b^{2}}+\frac{m_{2} \omega(b e-a f)}{a^{2}+b^{2}}\right\}
$$

Assume that the Fourier transform of the input ground acceleration is constant.

$$
\left|\ddot{U}_{g}(\omega)\right|=\bar{A}
$$

This input ground motion acceleration indicates the Dirac delta function at $t=0$ (Takewaki and Fujita 2009). Then the input energy can be related directly to the area of the energy transfer function and expressed as

$$
E_{I}=\bar{A}^{2} \int_{0}^{\infty} F_{C}(\omega) d \omega
$$

Since the energy given by the initial velocity is constant regardless of the stiffness and the damping coefficient of the model in the case of constant mass, it can be concluded that the area of the energy transfer function is constant regardless of the stiffness and the damping coefficient of the model.

\section{Earthquake Input Energy to Connected Models as Sum of Input Energies to Subassemblages}

While Eq.(12) defines the energy transfer function for the whole system, the energy transfer function can also be defined for each subassemblage. Assume $k_{3}=0$ here for simplicity. This means to deal with a viscous damper as the connecting system. 


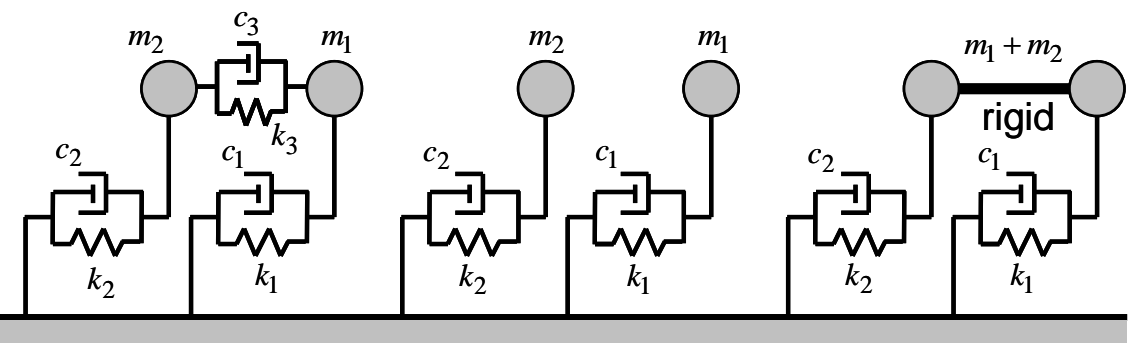

(a)

(b)

(c)

Fig.1 (a) Two SDOF models connected by viscous damper, (b) Disconnected two SDOF models, (c) Rigidly connected two SDOF models

Consider first the earthquake input energy in the time domain. The work done by the boundary forces in each subassemblage (building 1, building 2 and connecting damper) can be expressed as follows.

$$
\begin{aligned}
E_{I}^{1} /\left(m_{1}+m_{2}\right)= & \frac{1}{m_{1}+m_{2}} \int_{0}^{\infty}\left[c_{3}\left(\dot{u}_{2}-\dot{u}_{1}\right)\left(\dot{u}_{g}+\dot{u}_{1}\right)+\left(-k_{1} u_{1}-c_{1} \dot{u}_{1}\right) \dot{u}_{g}\right] d t \\
E_{I}^{2} /\left(m_{1}+m_{2}\right)= & \frac{1}{m_{1}+m_{2}} \int_{0}^{\infty}\left[-c_{3}\left(\dot{u}_{2}-\dot{u}_{1}\right)\left(\dot{u}_{g}+\dot{u}_{2}\right)+\left(-k_{2} u_{2}-c_{2} \dot{u}_{2}\right) \dot{u}_{g}\right] d t \\
& E_{I}^{3} /\left(m_{1}+m_{2}\right)=\frac{1}{m_{1}+m_{2}} \int_{0}^{\infty}\left[c_{3}\left(\dot{u}_{2}-\dot{u}_{1}\right)^{2}\right] d t
\end{aligned}
$$

Summation of Eqs.(15a-c), introduction of equilibrium equation between the boundary forces for the total system and the inertial force and use of integration by parts lead to the input energy to the overall system.

$$
E_{I} /\left(m_{1}+m_{2}\right)=-\frac{1}{m_{1}+m_{2}} \int_{0}^{\infty}\left(m_{1} \dot{u}_{1}+m_{2} \dot{u}_{2}\right) \ddot{u}_{g} d t
$$

This coincides with Eq.(12).

Fourier and inverse Fourier transformation of Eqs.(15a-c) lead to the following expression of the input energy in the frequency domain.

$$
E_{I}^{j} /\left(m_{1}+m_{2}\right)=\int_{0}^{\infty} F^{(j)}(\omega)\left|\ddot{U}_{g}(\omega)\right|^{2} d \omega(j=1,2,3)
$$

where

$$
\begin{aligned}
& F^{(1)}(\omega)=\frac{1}{\pi\left(m_{1}+m_{2}\right)} \operatorname{Re}\left[\omega^{2} c_{3}\left(H_{D 2}-H_{D 1}\right) H_{D 1}^{*}-c_{3}\left(H_{D 2}-H_{D 1}\right)+\frac{\mathrm{i}}{\omega}\left(-k_{1}-\mathrm{i} \omega c_{1}\right) H_{D 1}\right] \\
& F^{(2)}(\omega)=\frac{1}{\pi\left(m_{1}+m_{2}\right)} \operatorname{Re}\left[-\omega^{2} c_{3}\left(H_{D 2}-H_{D 1}\right) H_{D 2}^{*}+c_{3}\left(H_{D 2}-H_{D 1}\right)+\frac{\mathrm{i}}{\omega}\left(-k_{2}-\mathrm{i} \omega c_{2}\right) H_{D 2}\right]
\end{aligned}
$$




$$
F^{(3)}(\omega)=\frac{1}{\pi\left(m_{1}+m_{2}\right)} \operatorname{Re}\left[\omega^{2} c_{3}\left|H_{D 2}-H_{D 1}\right|^{2}\right]
$$

Eqs.(12), (16), (17) provide

$$
F_{C}(\omega)=F^{(1)}(\omega)+F^{(2)}(\omega)+F^{(3)}(\omega)
$$

The property of input energies to subassemblages can be understood from Eq. (17).

Consider the case of $k_{3}=0$. The masses are $m_{1}=m_{2}=32.0 \times 10^{3}(\mathrm{~kg})$. The spring stiffnesses are $k_{1}=1.88 \times 10^{7}(\mathrm{~N} / \mathrm{m})$ and $k_{2}=3.76 \times 10^{7}(\mathrm{~N} / \mathrm{m})$. The fundamental natural period of the main structure is $0.26(\mathrm{~s})$ and that of the substructure is $0.18(\mathrm{~s})$. This model corresponds to the case which is shown in the rightside figure in Fig.2 as a large dot (Mitsuda et al. 2014). The mass ratio is $\mu=m_{1} / m_{2}=1.0$ and the stiffness ratio is $\kappa=0.5$. In this case, the lowest mode represents the mode (mp-mode: meeting and parting-mode) in which both masses move in the reverse direction. In this model, the lowest damping ratio increases monotonically with respect to the damping coefficient of the connecting damper as shown in the left-side figure in Fig.2. The damping coefficients of the main structure and sub-structure are $c_{1}=1.88 \times 10^{5}(\mathrm{~N} \cdot \mathrm{s} / \mathrm{m}), c_{2}=3.76 \times 10^{5}(\mathrm{~N} \cdot \mathrm{s} / \mathrm{m})$. Three cases of damping coefficients of the connecting dampers are $c_{3}=3.76 \times 10^{5}(\mathrm{~N} \cdot \mathrm{s} / \mathrm{m}), 3.76 \times 10^{4}(\mathrm{~N} \cdot \mathrm{s} / \mathrm{m}), 3.76 \times 10^{3}(\mathrm{~N} \cdot \mathrm{s} / \mathrm{m})$. These values indicate the damping ratio $0.24,0.024,0.0024$ for the model with rigid sub-structure.

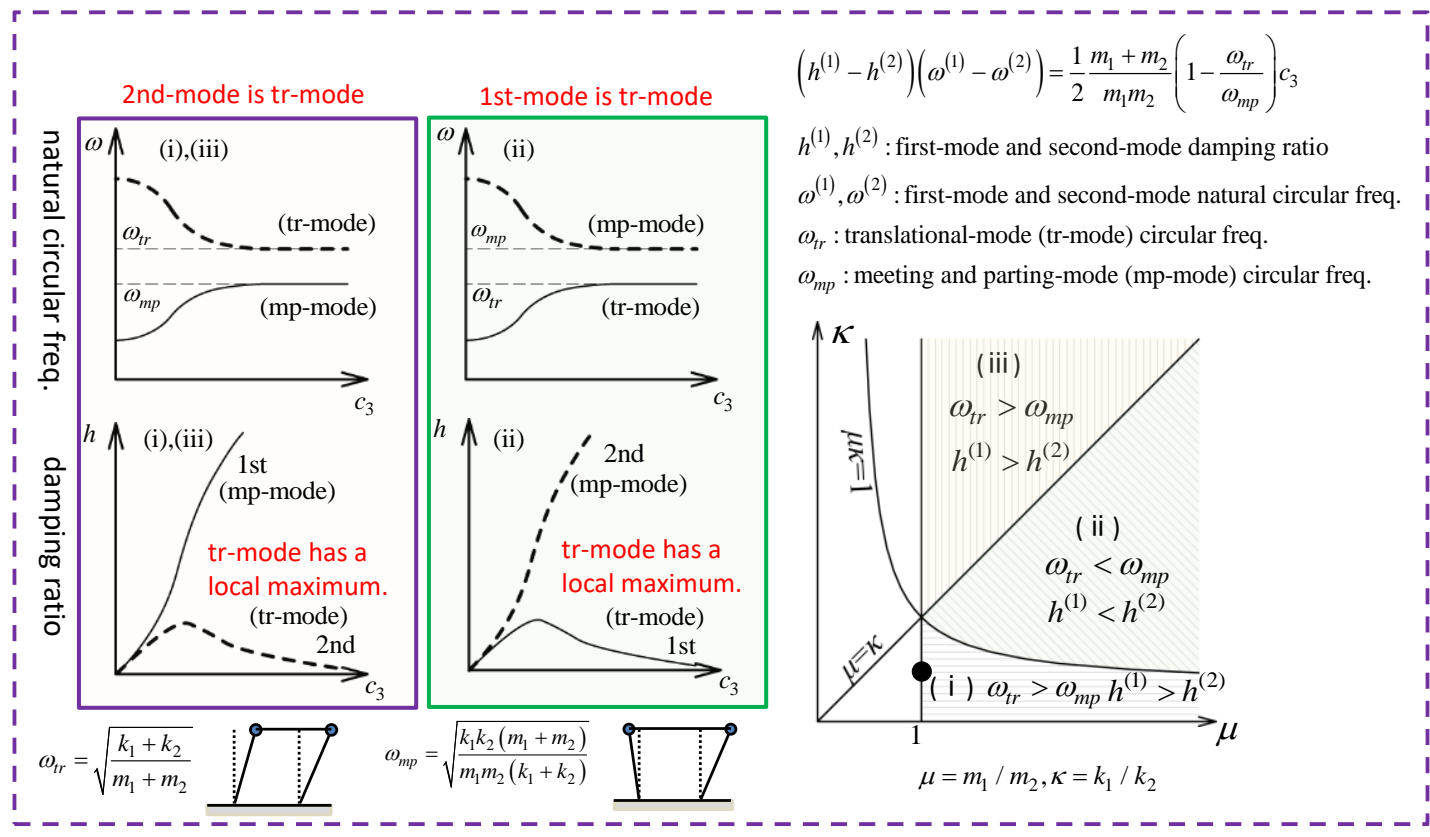

Fig.2 Classification of natural frequency and damping ratio properties with respect to connecting damper level 

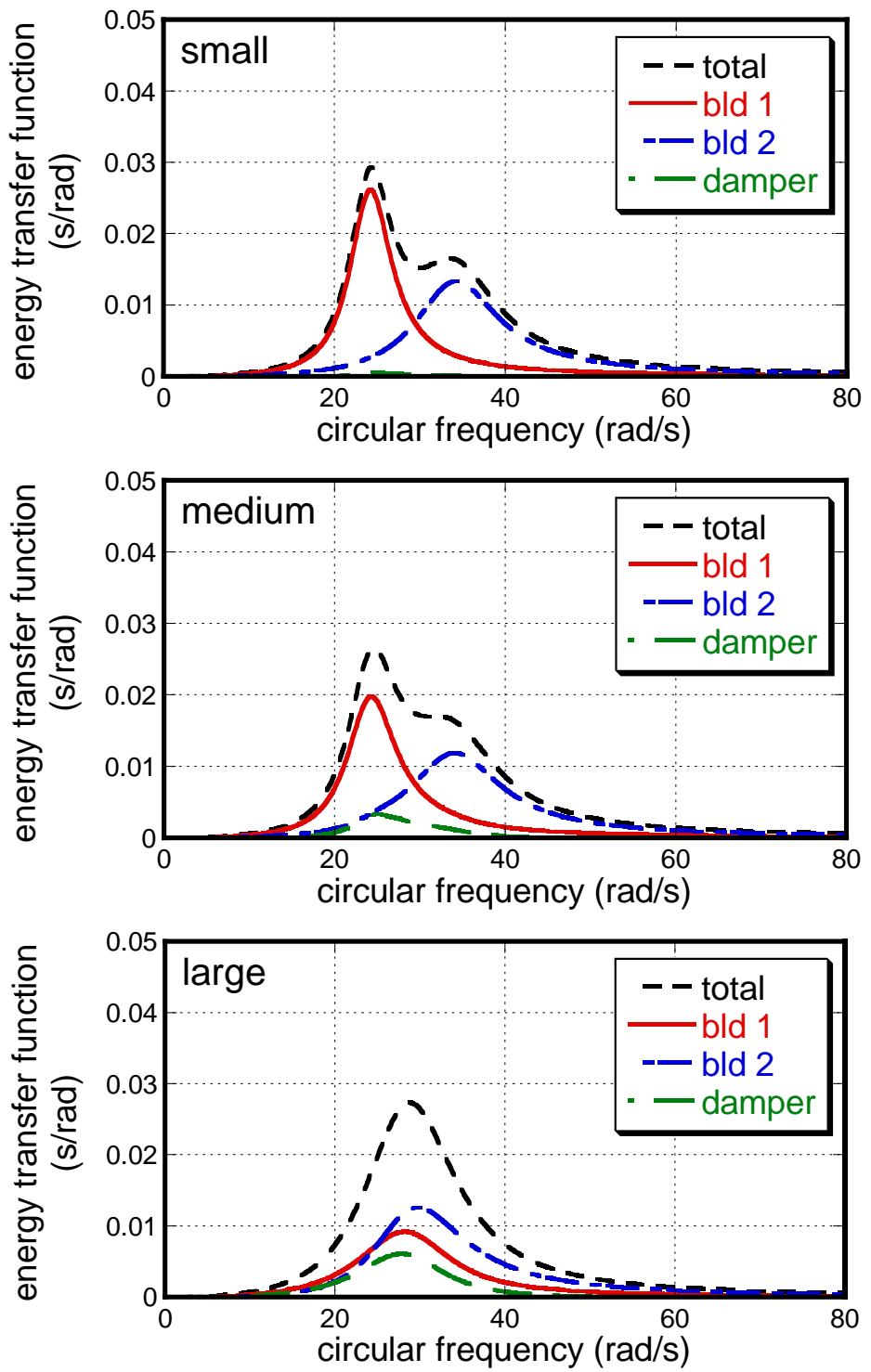

Fig.3 Energy transfer functions for total system and subassemblages for three levels of connecting damper

\section{Earthquake input energy by double impulse (Simplified impulsive ground motion)}

In Section 2-2, an input ground motion acceleration with a constant Fourier amplitude was considered. As pointed out before, this input ground motion acceleration is the Dirac delta function at $t=0$. A more practical input as a simplified impulsive ground motion may be a combination of impulses. It is well understood that one cycle sinusoidal motion is a good substitute of a class of near-fault ground motions (Fling-step input) (see Mavroeidis and Papageorgiou 2003, Kalkan and Kunnath 2006, Khaloo et al. 2015). It can be confirmed that, if the Fourier amplitudes of the double impulse and one cycle sinusoidal motion coincide well, the 
maximum displacement responses of a resonant undamped single-degree-of-freedom system under both input models exhibit a good agreement. This comparison is shown in Fig.4 where $d_{y}$ is a reference deformation and $V_{y}$ is a reference input level.

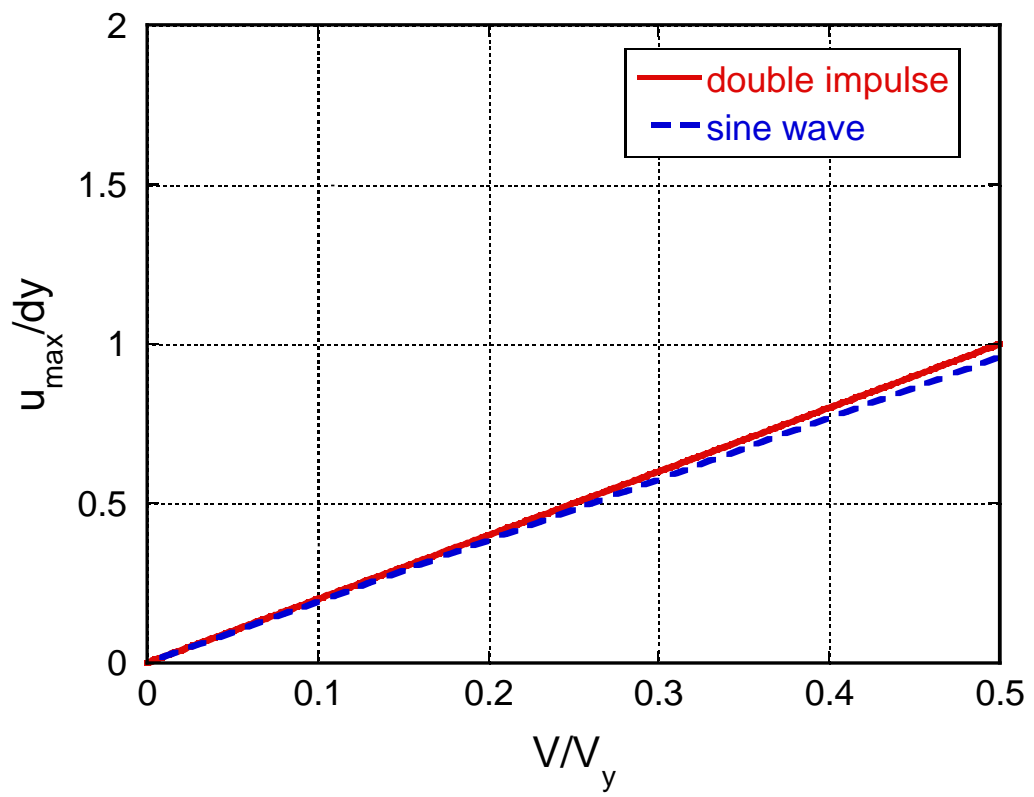

Fig.4 Comparison of the maximum deformation of a resonant one-degree-offreedom system under double impulse and the corresponding one-cycle sinusoidal wave

Consider the following double impulse with $t_{0}$ as the interval (see Figs.5, 6).

$$
\ddot{u}_{g}(t)=V \delta(t)-V \delta\left(t-t_{0}\right)
$$

where $V$ is the change of velocity of ground motion at $t=0$ and $t=t_{0}$.

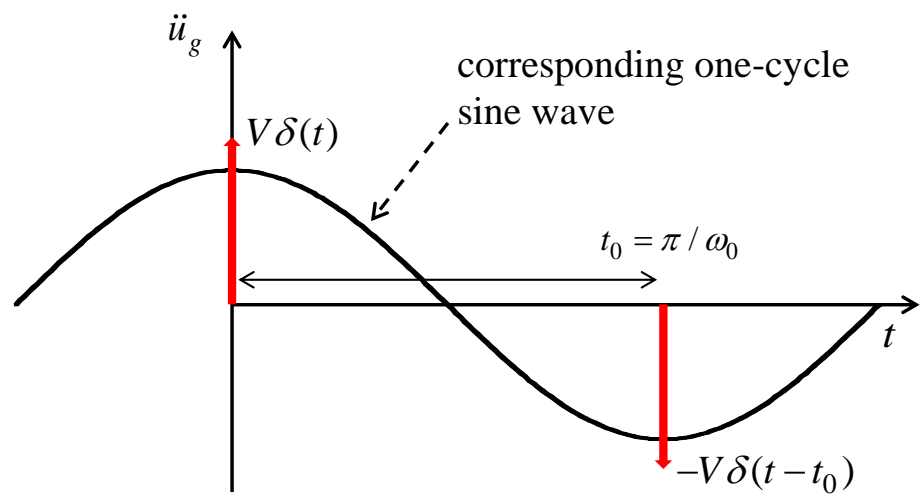

Fig.5 Double impulse as simplification of impulsive ground motion acceleration 


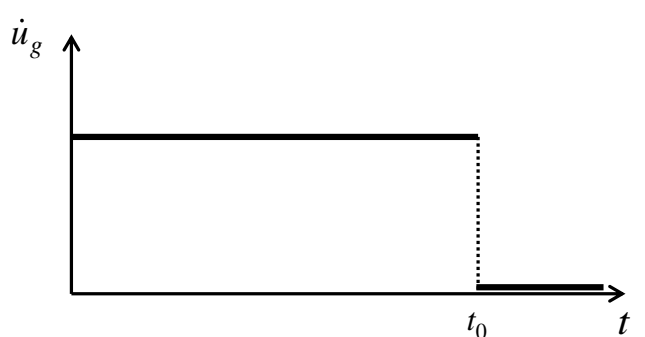

(a)

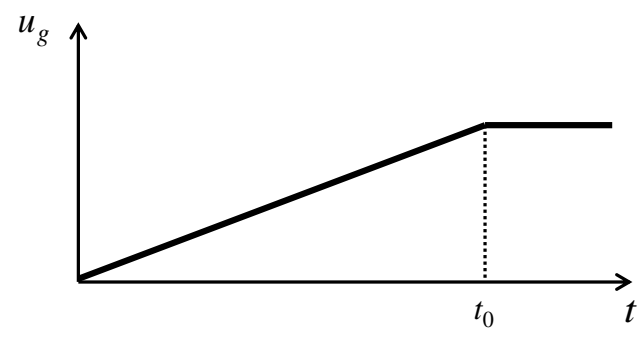

(b)

Fig.6 Velocity and displacement of double impulse

The Fourier transform of Eq.(20) is expressed as

$$
\begin{aligned}
\ddot{U}_{g}(\omega) & =\int_{-\infty}^{\infty}\left\{V \delta(t)-V \delta\left(t-t_{0}\right)\right\} e^{-\mathrm{i} \omega t} d t \\
& =\int_{-\infty}^{\infty}\left\{V \delta(t) e^{-\mathrm{i} \omega t}-V \delta\left(t-t_{0}\right) e^{-\mathrm{i} \omega t_{0}} e^{-\mathrm{i} \omega\left(t-t_{0}\right)}\right\} d t \\
& =V\left(1-e^{-\mathrm{i} \omega t_{0}}\right)
\end{aligned}
$$

The square of the absolute value of Eq.(21) is derived as

$$
\left|\ddot{U}_{g}(\omega)\right|^{2}=V^{2}\left(2-2 \cos \omega t_{0}\right)
$$

The normalized squared Fourier amplitude $2-2 \cos \omega t_{0}$ of the double impulse is shown in Fig.7 $\left(t_{0}=1 \mathrm{~s}\right)$ together with the corresponding sine wave of one cycle. It can be found that a fairly good correspondence exists in the first peak. Because the first peak plays an important role in the evaluation of the critical input as explained just afterward, this correspondence in the first cycle is sufficient for the simplification of the sinusoidal wave (impulsive ground motion) by double impulse.

The validity of using the double impulse as a substitute of one-cycle sine wave as shown in Fig.5 can be demonstrated by using time-history response analyses. The result is presented in Appendix 1. It can be concluded that the double impulse is a good substitute of the one-cycle sine wave so long as their maximum Fourier amplitudes coincide.

Eq.(12) for this input is obtained as

$$
E_{I} /\left(m_{1}+m_{2}\right)=\int_{0}^{\infty} F_{C}(\omega) V^{2}\left(2-2 \cos \omega t_{0}\right) \mathrm{d} \omega
$$

The normalized expression of Eq.(23) can be described as

$$
E_{I} /\left\{\left(m_{1}+m_{2}\right) V^{2}\right\}=\int_{0}^{\infty} F_{C}(\omega)\left(2-2 \cos \omega t_{0}\right) \mathrm{d} \omega
$$

A critical excitation problem (Drenick 1970, Takewaki 2001, 2002, 2007a) can be formulated as follows for this double impulse. 
[Critical Excitation Problem 1] Find $t_{0}$ of double impulse so as to maximize the normalized earthquake input energy $E_{I} /\left\{\left(m_{1}+m_{2}\right) V^{2}\right\}$ given by Eq.(24).

The worst interval of these impulses can be defined as such that the input energy attains the maximum (see Fig.8).

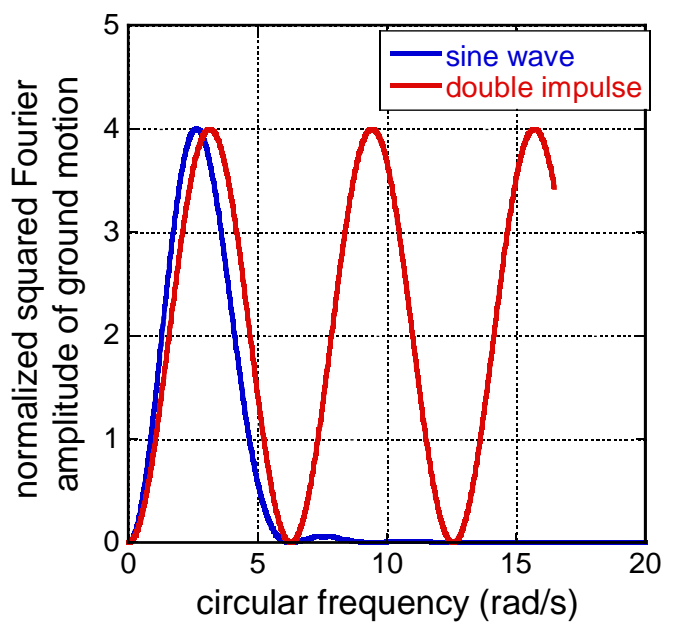

Fig.7 Normalized squared Fourier amplitude of ground motion as double impulse and its comparison with that of the corresponding sine wave of one cycle (the maximum value is adjusted)

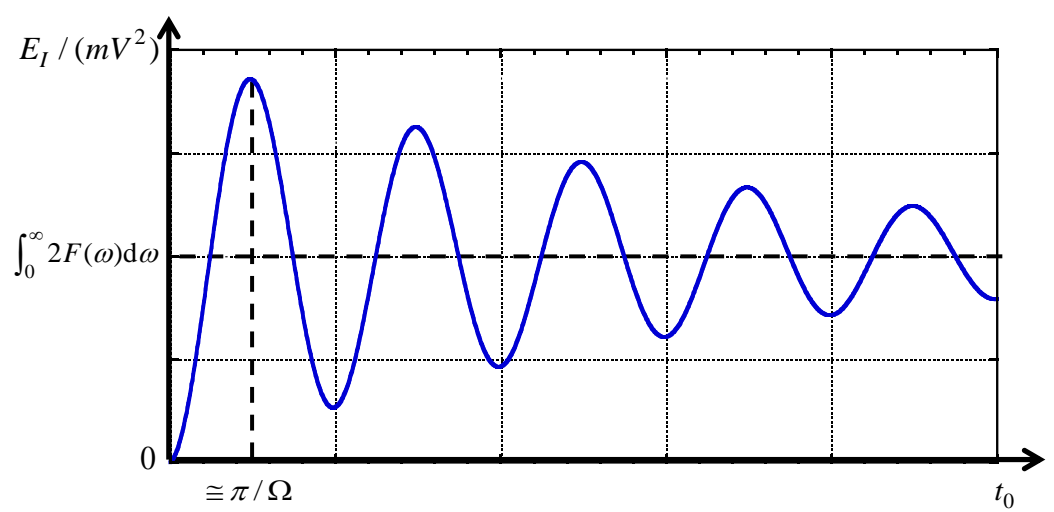

Fig.8 Schematic diagram of normalized earthquake input energy with respect to interval of double impulse

The normalized input energies to subassemblies can be expressed following Eq.(17).

$$
E_{I}^{j} /\left\{\left(m_{1}+m_{2}\right) V^{2}\right\}=\int_{0}^{\infty} F^{(j)}(\omega)\left(2-2 \cos \omega t_{0}\right) d \omega(j=1,2,3)
$$

The worst interval of impulses can also be defined for each subassemblage. 


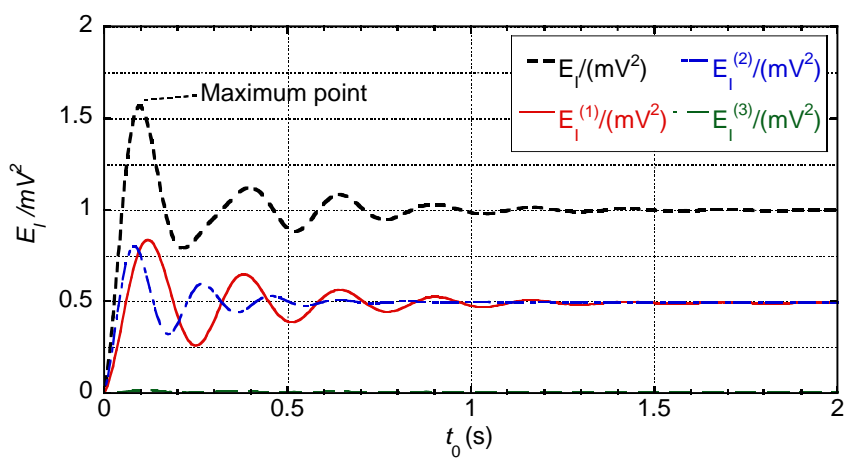

Connecting damper level: small

Fig.9 Input energies to overall system, main structure, substructure and connecting damper with respect to interval of double impulses

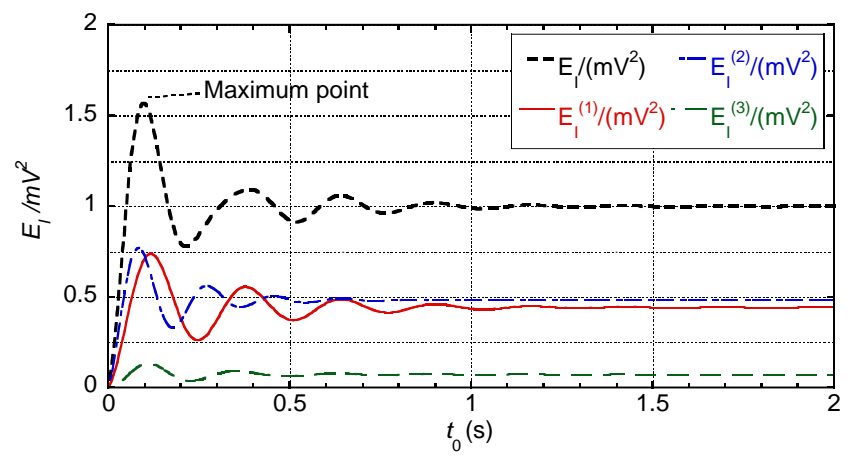

Connecting damper level: medium

Fig.10 Input energies to overall system, main structure, substructure and connecting damper with respect to interval of double impulses

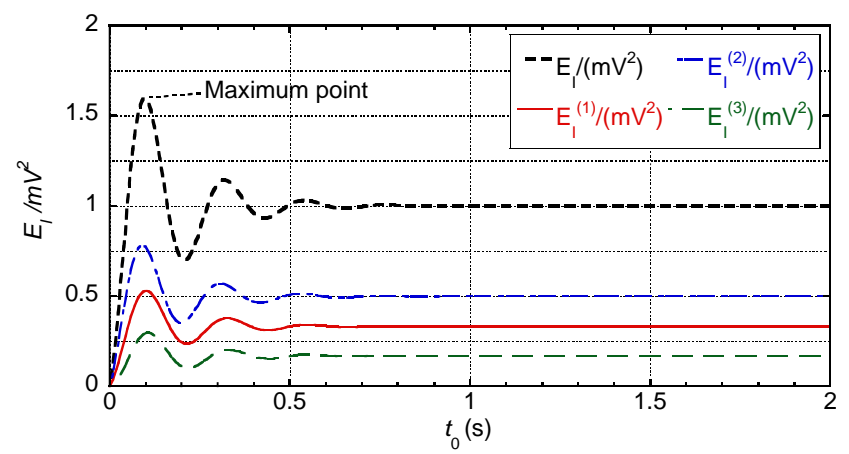

Connecting damper level: large

Fig.11 Input energies to overall system, main structure, substructure and connecting damper with respect to interval of double impulses

Figs.9-11 show the input energies, defined by Eqs.(24), (25), to the overall system, the main structure, the substructure and the connecting damper with respect to $t_{0}$ for three damper levels (small, medium, large). It can be observed that the worst interval of two impulses can be different for the main structure, the substructure and the connecting damper depending on the connecting damper levels (small, medium, large). 


\section{Earthquake input energy by multiple impulses (Simplified long-duration ground motion)}

The double impulse represents an impulsive type earthquake ground motion. On the other hand, the long-duration ground motion can be represented by the following $N$ impulses (see Fig.12).

$$
\ddot{u}_{g}(t)=V \delta(t)-V \delta\left(t-t_{0}\right)+V \delta\left(t-2 t_{0}\right)-V \delta\left(t-3 t_{0}\right)+\cdots
$$

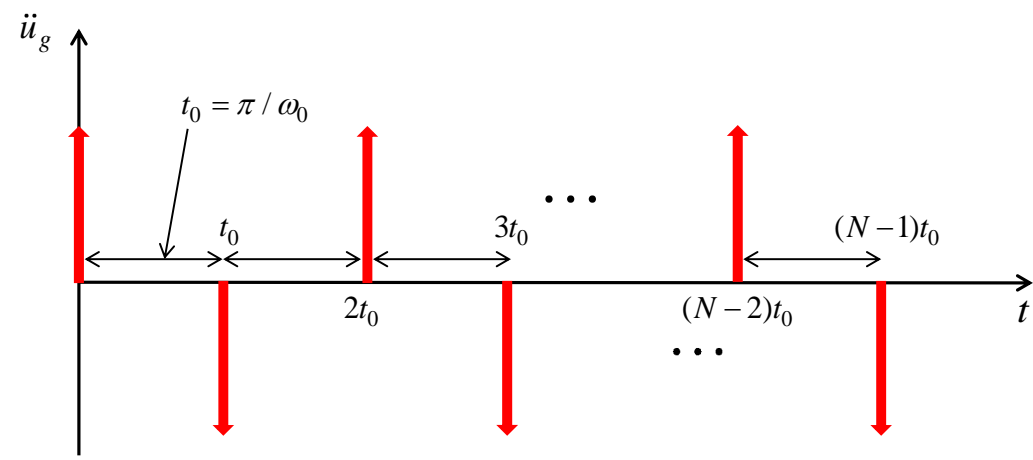

Fig.12 $N$ impulses as simplification of long-duration ground acceleration

The Fourier transform of Eq.(26) can be obtained as

$$
\begin{aligned}
\ddot{U}_{g}(\omega)= & \int_{-\infty}^{\infty}\left\{V \delta(t)-V \delta\left(t-t_{0}\right)+V \delta\left(t-2 t_{0}\right)-V \delta\left(t-3 t_{0}\right)+\cdots\right\} e^{-\mathrm{i} \omega t} d t \\
= & \int_{-\infty}^{\infty}\left\{V \delta(t) e^{-\mathrm{i} \omega t}-V \delta\left(t-t_{0}\right) e^{-\mathrm{i} \omega t_{0}} e^{-\mathrm{i} \omega\left(t-t_{0}\right)}\right. \\
& \left.+V \delta\left(t-2 t_{0}\right) e^{-\mathrm{i} \omega 2 t_{0}} e^{-\mathrm{i} \omega\left(t-2 t_{0}\right)}-V \delta\left(t-3 t_{0}\right) e^{-\mathrm{i} \omega 3 t_{0}} e^{-\mathrm{i} \omega\left(t-3 t_{0}\right)}+\cdots\right\} d t \\
= & V\left(1-e^{-\mathrm{i} \omega t_{0}}+e^{-\mathrm{i} \omega 2 t_{0}}-e^{-\mathrm{i} \omega 3 t_{0}}+\cdots\right)
\end{aligned}
$$

The square of the absolute value of Eq.(27) leads to

$$
\left|\ddot{U}_{g}(\omega)\right|^{2}=V^{2}\left|1-e^{-\mathrm{i} \omega t_{0}}+e^{-\mathrm{i} \omega 2 t_{0}}-e^{-\mathrm{i} \omega 3 t_{0}}+\cdots\right|^{2}=V^{2}\left|1+\sum_{n=1}^{N-1}(-1)^{n} e^{-\mathrm{i} \omega n t_{0}}\right|^{2}
$$

The normalized squared Fourier amplitude spectrum $\left|1+\sum_{n=1}^{N-1}(-1)^{n} e^{-i \omega n t_{0}}\right|^{2}$ for 20 impulses $\left(t_{0}=1.0 \mathrm{~s}\right)$ is shown in Fig.13 together with that for the corresponding sinusoidal wave of 10 cycles. It can be observed that a good coincidence exists in the first peak. Since only the first peak plays an important role in the evaluation of the critical input as in the case of the double impulse, this correspondence in the first cycle is sufficient for the simplification of the sinusoidal wave (long-duration ground motion) by 20 impulses. When we compute the input energy by 20 impulses $(N=20)$, it is sufficient to replace $\left(2-2 \cos \omega t_{0}\right)$ in Eq.(24), (25) with Eq.(28). The resulting equation may be expressed as

$$
E_{I} /\left\{\left(m_{1}+m_{2}\right) V^{2}\right\}=\int_{0}^{\infty} F_{C}(\omega)\left|1+\sum_{n=1}^{N-1}(-1)^{n} e^{-\mathrm{i} \omega n t_{0}}\right|^{2} \mathrm{~d} \omega
$$




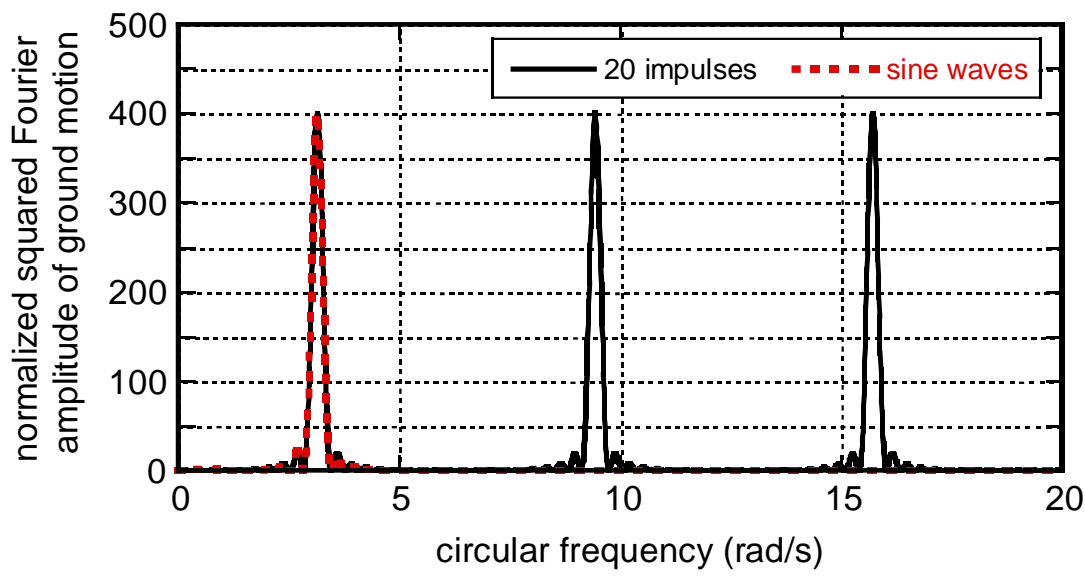

Fig.13 Normalized squared Fourier amplitude of ground motion as 20 impulses and its comparison with that of the corresponding sine wave of 10 cycles (the maximum value is adjusted)

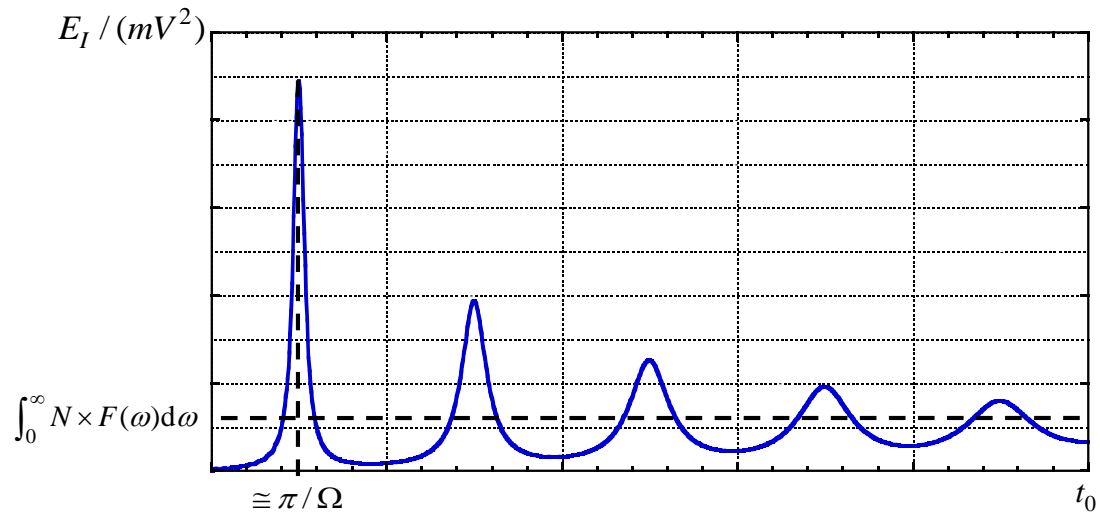

Fig.14 Schematic diagram of normalized earthquake input energy with respect to interval of 20 impulses

A schematic diagram of normalized earthquake input energy with respect to the interval of 20 impulses is shown in Fig.14. It may be possible to formulate a critical excitation problem as follows with the interval of impulses as a variable.

[Critical Excitation Problem 2] Find $t_{0}$ of multiple impulses so as to maximize the normalized earthquake input energy $E_{I} /\left\{\left(m_{1}+m_{2}\right) V^{2}\right\}$ given by Eq.(29).

Figs.15-17 show the normalized earthquake input energies to the overall system and each subsystem with respect to impulse interval for three levels of connecting dampers. The model parameters are the same as the above case. The worst interval of impulses can also be defined for each subassemblage. It can be observed that, when the level of the connecting dampers is small, the building 1 (main structure), building 2 (substructure), connecting dampers and the total system exhibit different critical intervals of impulses. On the other hand, when the level of the connecting dampers is large, those exhibit almost the same critical interval. It can also be understood that, as $t_{0}$ becomes large, the normalized earthquake input energy converges to $\int_{0}^{\infty} N \times F(\omega) d \omega$. 


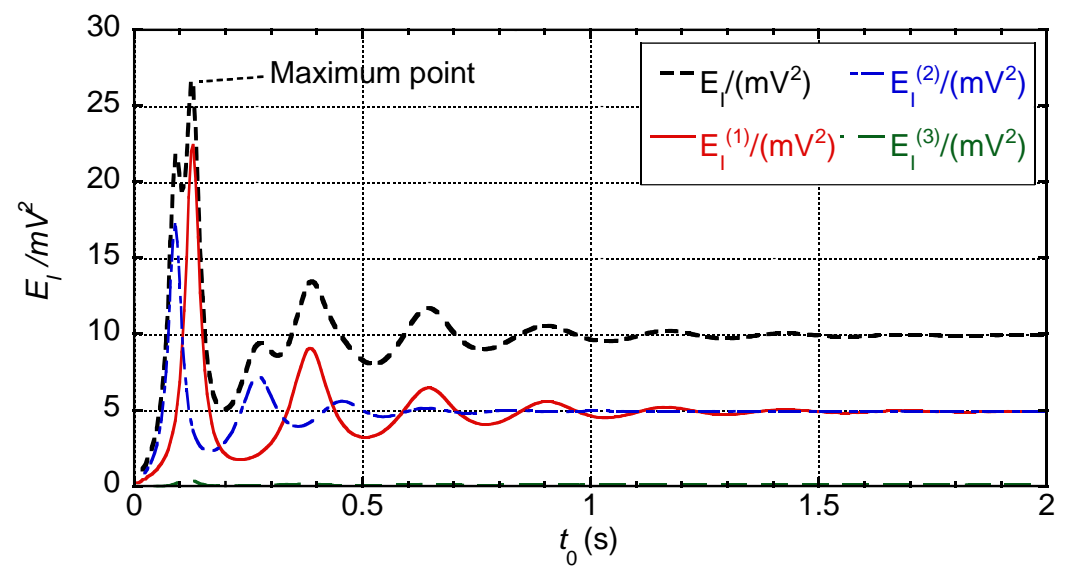

Connecting damper level: small

Fig.15 Input energies to overall system, main structure, substructure and connecting damper with respect to interval of 20 impulses

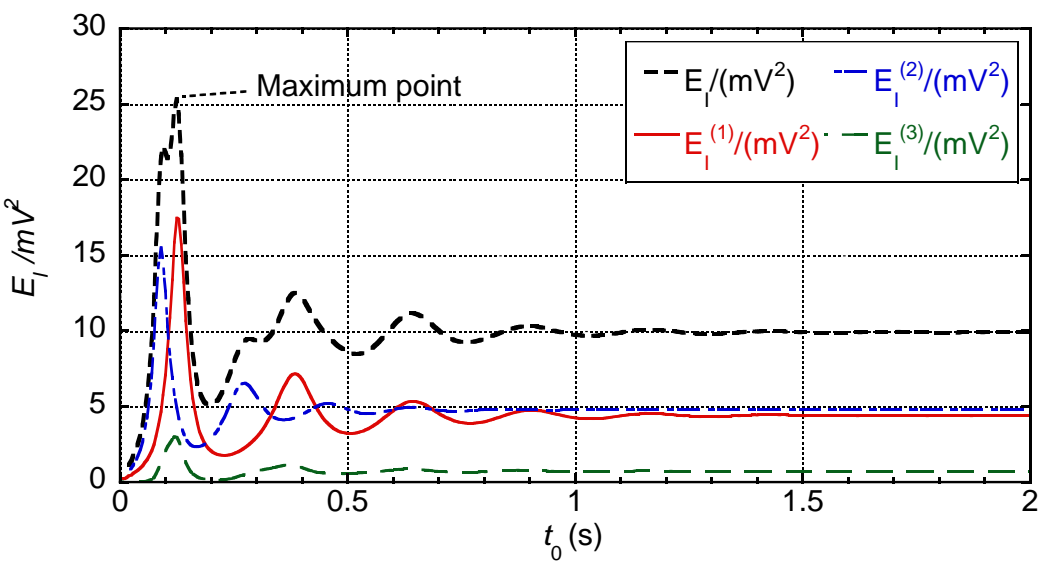

Connecting damper level: medium

Fig.16 Input energies to overall system, main structure, substructure and connecting damper with respect to interval of 20 impulses

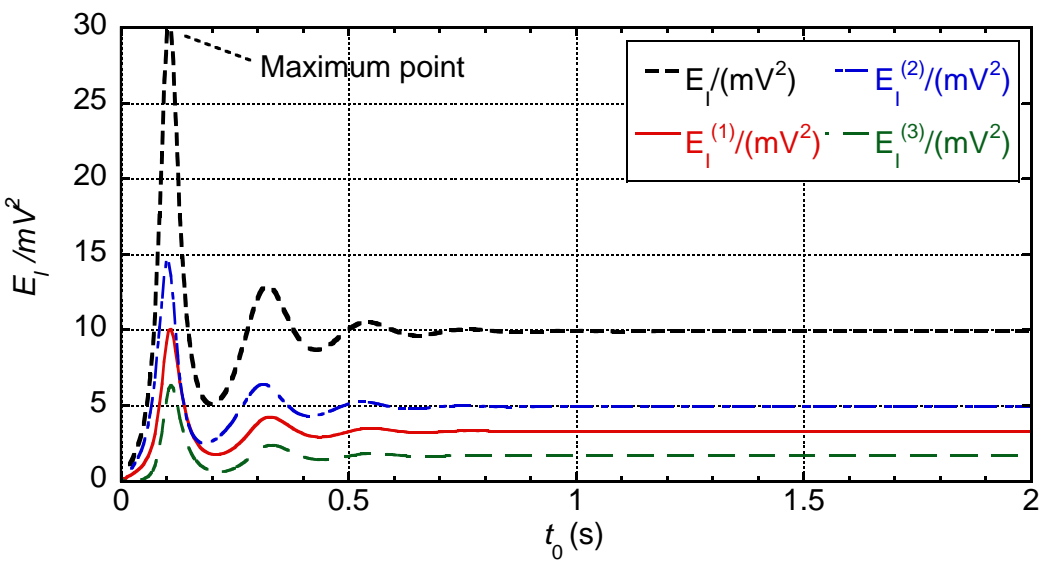

Connecting damper level: large

Fig.17 Input energies to overall system, main structure, substructure and connecting damper with respect to interval of 20 impulses 


\section{Conclusions}

The conclusions may be stated as follows.

(1) The energy transfer function $F(\omega)$ of damper-connected building models characterizing the earthquake input energy in the frequency domain has an equiarea property regardless of the connecting damper quantity. This property of $F(\omega)$ can be proved by the time-domain method for an idealized model of input motions with a constant Fourier amplitude spectrum. This idealized input model corresponds to the acceleration of the Dirac delta function in the time domain and the input energy can be characterized by the initially given kinetic energy depending only on the total mass and the initial velocity.

(2) The proposed energy transfer functions for the overall system and each subassemblage (respective buildings and connecting viscous dampers) are very useful for clear understanding of dependence of energy consumption ratios in respective buildings and connecting viscous dampers on their properties.

(3) Critical excitation problems with respect to the impulse interval for double impulse (simplification of pulse-type impulsive ground motion) and multiple impulses (simplification of long-duration ground motion) have been considered and their solutions have been provided. The frequency domain formulation of earthquake input energy enabled the direct solution to the critical excitation problem. It has been made clear that the worst interval of two or multiple impulses can be different for the main structure, the substructure and the connecting damper depending on the connecting damper levels.

It is anticipated that the input energy to the overall system by a general ground motion input is approximately constant regardless of the quantity of connecting viscous dampers. This property is also guaranteed and supported by the equi-area property of the energy transfer function and leads to an advantageous feature that, if the energy consumption in the connecting dampers increases, the input energies to the buildings can be reduced effectively.

The evaluation of the earthquake input energy in the time domain is suitable for the evaluation of the time history of the input energy, especially for non-linear systems. Dual use of the frequency-domain and time-domain techniques may be preferable in the advanced seismic analysis for robuster design.

\section{Acknowledgements}

Part of the present work is supported by the Grant-in-Aid for Scientific Research of Japan Society for the Promotion of Science (No.24246095, 15H04079). This support is greatly appreciated. Part of the numerical computation was conducted by Mr. K. Kojima of Kyoto University. The authors are grateful to him. 


\section{References}

Akiyama, H. (1985). Earthquake Resistant Limit-State Design for Buildings. University of Tokyo Press, Tokyo, Japan.

Basili M, Angelis MD. (2007). Optimal passive control of adjacent structures interconnected with nonlinear hysteretic devices. Journal of Sound and Vibration; 301(1-2):106-125.

Christenson RE, Spencer BF, Johnson EA.(2007). Semiactive connected control method for adjacent multi-degree-of freedom buildings. Journal of Engineering Mechanics, ASCE; 133(3):290-298.

Cimellaro, GP. and Lopez-Garcia, D. (2011). Algorithm for design of controlled motion of adjacent structures. Journal of Structural Control and Health Monitoring; 18, 140-148.

Drenick, RF. (1970). Model-free design of aseismic structures. Journal of the Engineering Mechanics Division, ASCE; 96(EM4), 483-493.

Housner, G.W. (1959). Behavior of structures during earthquakes. Journal of the Engineering Mechanics Division, ASCE, 85(4), 109-129.

Kalkan, E. and Kunnath, S.K. (2006). Effects of fling step and forward directivity on seismic response of buildings, Earthquake Spectra, 22(2), 367-390.

Khaloo, A.R., Khosravi1, H. and Hamidi Jamnani, H. (2015). Nonlinear interstory drift contours for idealized forward directivity pulses using "Modified FishBone" models; Advances in Structural Eng.18(5), 603-627.

Kim K, Rye J, Chung L. (2006). Seismic performance of structures connected by viscoelastic dampers. Engineering Structures; 28(2):83-195.

Kuwamura, H., Kirino, Y., and Akiyama, H. (1994). Prediction of earthquake energy input from smoothed Fourier amplitude spectrum. Earthquake Engineering and Structural Dynamics, 23, 1125-1137.

Leger, P., and Dussault, S. (1992). Seismic-energy dissipation in MDOF structures. Journal of Structural Engineering, ASCE, 118(5) 1251-1269.

Luco JE and Barros FCP. (1998). Optimal damping between two adjacent elastic structures. Earthquake Engineering and Structural Dynamics; 27(7):649-659.

Lyon, R.H. (1975). Statistical Energy Analysis of Dynamical Systems, The MIT Press, Cambridge, MA.

Mavroeidis, G. P., and Papageorgiou, A. S. (2003). A mathematical representation of near-fault ground motions, Bull. Seism. Soc. Am., 93(3), 1099-1131.

Mitsuda, E., Ohbuchi, M., Tsuji M. and Takewaki, I. (2014). Fundamental properties on eigenvibration and damping in connected building structures, $J$. Construction and Structural Engineering, Archi. Inst. of Japan, No.696, 227236 (in Japanese).

Murase, M., Tsuji, M. and Takewaki, I. (2013). Smart passive control of buildings with higher redundancy and robustness using base-isolation and interconnection, Earthquakes and Structures, 4(6), 649-670.

Ok S, Song J, Park K. (2008). Optimal design of hysteretic dampers connecting adjacent structures using multiobjective genetic algorithm and stochastic linearization method. Engineering Structures; 30(5):1240-1249. 
Ordaz, M., Huerta, B., and Reinoso, E. (2003). Exact computation of input-energy spectra from Fourier amplitude spectra. Earthquake Engineering and Structural Dynamics, 32, 597-605.

Richardson, A. , Walsh, K. K. and Abdullah, M. M. (2013a). Closed-Form Design Equations for Controlling Vibrations in Connected Structures, Journal of Earthquake Engineering, 17:699-719.

Richardson, A., Walsh, K. K., and Abdullah, M. M. (2013b). Closed-form equations for coupling linear structures using stiffness and damping elements, Journal of Structural Control and Health Monitoring 20(3), 259-281.

Roh, H., Cimmellaro, GP. and Lopez-Garcia, D. (2011). Seismic Response of Adjacent Steel Structures Connected by Passive Device. 14(3), 499-517, Advances in Structural Engineering.

Seto K. (1994). Vibration control method for flexible structures arranged in parallel. Proceedings of the 1st World Conference on Structural Control, vol. 2, Pasadena, California; 62-71.

Takewaki, I. (2001). A new method for nonstationary random critical excitation. Earthquake Engineering and Structural Dynamics, 30(4), 519-535.

Takewaki, I. (2002). Critical excitation method for robust design: A review. Journal of Structural Engineering, ASCE, 128(5), 665-672.

Takewaki, I. (2004a). Bound of earthquake input energy, Journal of Structural Engineering, ASCE, 130(9), 1289-1297.

Takewaki, I. (2004b). Frequency domain modal analysis of earthquake input energy to highly damped passive control structures, Earthquake Engineering and Structural Dynamics; 33(5): 575-590.

Takewaki, I. (2005a). Frequency domain analysis of earthquake input energy to structure-pile systems, Engineering Structures, 27(4), 549-563.

Takewaki, I. (2005b). Bound of earthquake input energy to soil-structure interaction systems, Soil Dynamics and Earthquake Engineering, 25(7-10), 741-752.

Takewaki, I. (2007a). Critical excitation methods in earthquake engineering, Elsevier, Second edition in 2013.

Takewaki, I. (2007b). Earthquake input energy to two buildings connected by viscous dampers, Journal of Structural Engineering, ASCE, 133(5), 620-628.

Takewaki, I., and Fujita, K. (2009). Earthquake input energy to tall and base-isolated buildings in time and frequency dual domains, J. of The Structural Design of Tall and Special Buildings; 18(6): 589-606.

Uang, C.M., and Bertero, V.V. (1990). Evaluation of seismic energy in structures. Earthquake Engineering and Structural Dynamics, 19, 77-90.

Xu YL, He Q and Ko JM. (1999). Dynamic response of damper-connected adjacent buildings under earthquake excitation. Engineering Structures; 21:135-148.

Zahrah, T.F., and Hall, W.J. (1984). Earthquake energy absorption in SDOF structures. Journal of Structural Engineering, ASCE, 110(8) 1757-1772.

Zhu HP, Ge DD, Huang X. (2011). Optimum connecting dampers to reduce the seismic responses of parallel structures. Journal of Sound and Vibration; 330(9):1931-1949. 


\section{Appendix 1: Accuracy of double impulse as a substitute of one-cycle sine wave}

Consider the same connected building model as shown in Section 3. The damping coefficient of the connecting damper is $c_{3}=3.76 \times 10^{4}(\mathrm{~N} \cdot \mathrm{s} / \mathrm{m})$ (medium). Fig.A1 shows the displacement time histories of the main structure and the substructure in the case where $t_{0}$ (interval of double impulses) is half the natural period of the 'main structure'. It can be observed that the response to the double impulse can be a good substitute of that to the corresponding one-cycle sine wave. On the other hand, Fig.A2 presents the displacement time histories of the main structure and the substructure in the case where $t_{0}$ is half the natural period of the 'substructure'. It can be observed again that the response to the double impulse can be a good substitute of that to the corresponding one-cycle sine wave. Furthermore it can be understood from these figures that the responses of the structure (main structure or substructure) to both inputs exhibit better correspondence when the natural period of that structure coincides with twice of the input interval $t_{0}$.

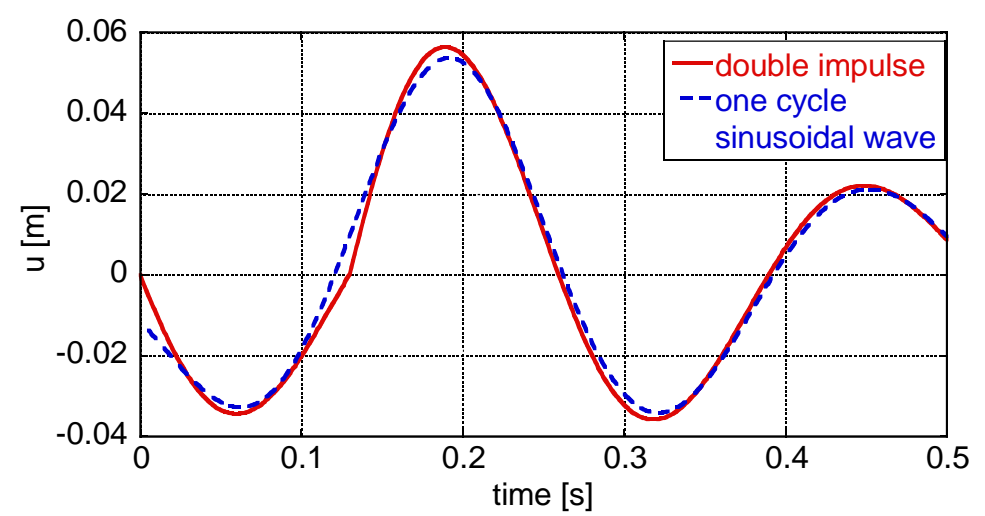

(a) Response of main structure

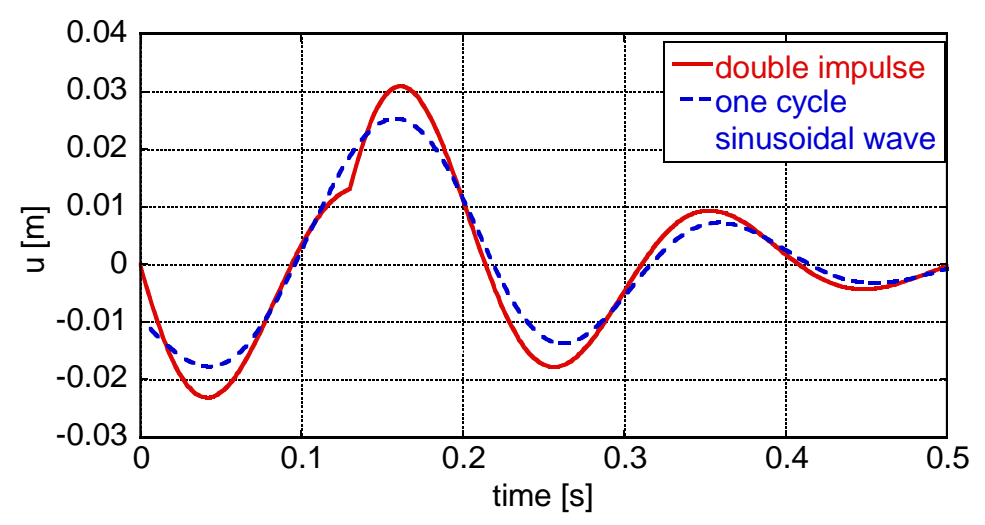

(b) Response of substructure

Fig.A1 Displacement time histories of the main structure and the substructure in the case where $t_{0}$ (interval of double impulses) is half the natural period of the main structure 


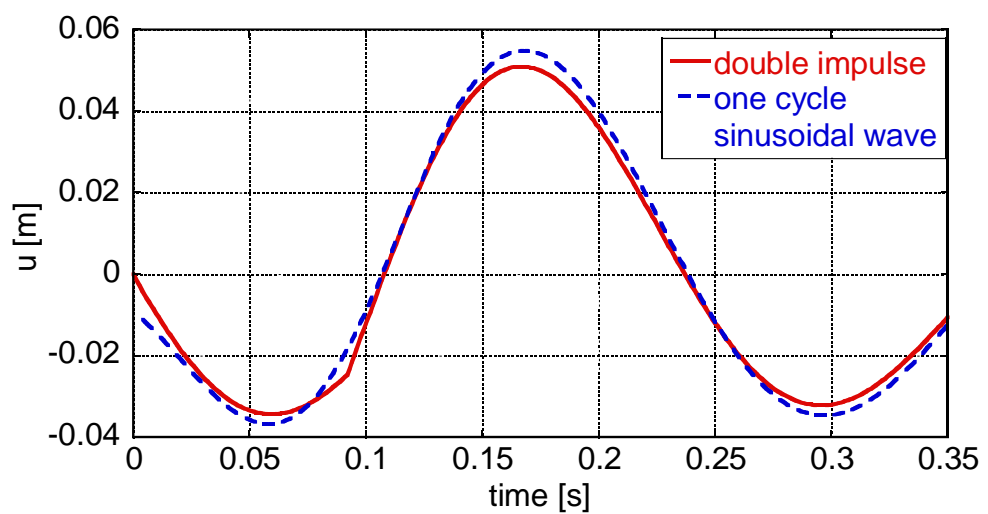

(a) Response of main structure

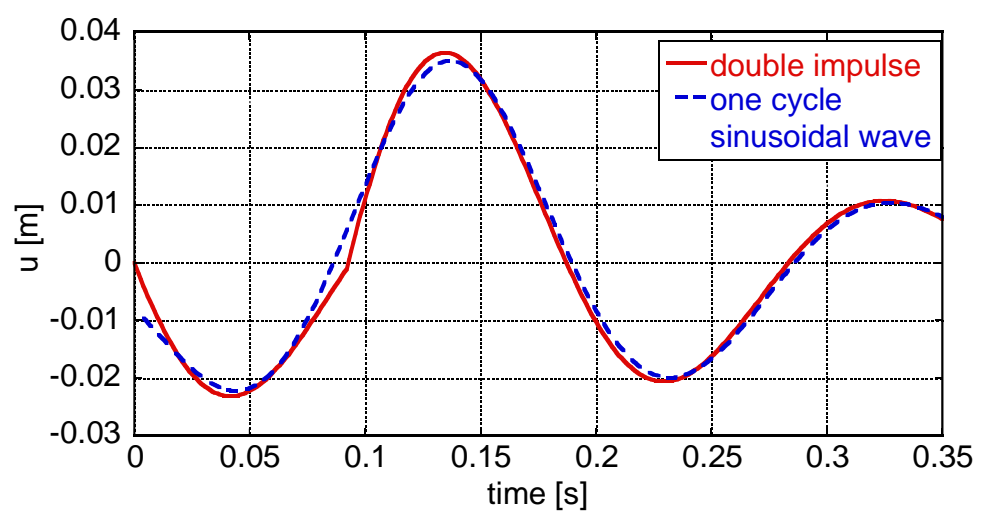

(b) Response of substructure

Fig.A2 Displacement time histories of the main structure and the substructure in the case where $t_{0}$ (interval of double impulses) is half the natural period of the substructure 